\title{
A unique Anatomical variation of flexor digitorum superficialis muscle: a cadaveric case report
}

\author{
Udhayakumar $\mathrm{S}^{1}$, Nilojan $\mathrm{JS}^{1}$, Ketheeswaran $\mathrm{N}^{1}$, Srishankar $\mathrm{S}^{1}$ \\ ${ }^{1}$ Department of Anatomy, Faculty of Medicine, University of Jaffna, Sri Lanka
}

Running title: Variation of flexor digitorum superficialis

\begin{abstract}
Anatomical variations of the flexor digitorum superficialis (FDS) muscle are uncommon and is related to evolution of muscle from amphibians to human. These variations are of academic interest and should be considered when facing variations in clinical examination, interpreting radiological images and in surgical procedures such as tendon graft.
\end{abstract}

We report a case of unilateral variation of FDS muscle in the forearm with two muscle bellies: superficial and deep which has not been reported before. The humeroulnar and radial origins of the superficial belly united to form a single muscle belly, which divided in the upper part of forearm into two tendons, that passed through carpal tunnel and inserted into the middle phalanges of third and fourth digits. The deep belly originated from medial epicondyle of the humerus next to flexor carpi ulnaris, continued as a short tendon in the upper part of forearm and then formed a second muscle belly in the middle third of the forearm (two muscle bellies separated by an intermediate tendon). In the lower part of forearm deep muscle belly divided into two tendons which traversed the carpal tunnel and was inserted into middle phalanx of second and fifth digits.

The report enhances the knowledge on variation of FDS and add a note to understand atypical findings on physical examination and during surgery.

Key words: Anatomical variation, forearm flexors, Flexor digitorum superficialis, carpal tunnel

\section{Introduction}

The flexor digitorum superficialis (FDS) is an extrinsic muscle of the hand. It arises from two heads - humeroulnar and radial, which unite to form a singular muscle belly in the forearm between the superficial and deep muscle groups. In the lower part of antebrachium, it splits into four tendon. The tendons pass deep to the flexor retinaculum within the carpal tunnel in 2 rows, with the tendons for middle and ring finger being superficial to that of index and little finger. The tendons diverge towards the medial four digits and enters the digital flexor sheath. At the base of the proximal phalanx each tendon splits into 2 slips to allow the passage of flexor digitorum profundus (FDP) and are inserted into the sides of the shaft of middle phalanx of the 
Udhayakumar S, Nilojan JS, Ketheeswaran N, Srishankar S - A unique Anatomical variation of flexor digitorum superficialis muscle: a cadaveric case report

corresponding digit. The FDS is a prime flexor of proximal interphalangeal joint (PIPJ) (1).

Anatomical discrepancy of FDS muscle belly, abnormal connections of muscle and tendon and absence of the tendinous part have been described in anatomical and clinical studies. Most of them are cadaveric findings (2, 3, 4, 5, $6,7,8)$. Some are incidental asymptomatic finding during surgery $(9,10,11)$. Palm muscle mass resembling soft tissue tumor (10, $12,13,14)$ and carpal tunnel syndrome due to undetermined aetiology 10, 12, 13) are rare symptomatic findings. Elliot et al (10) classified variations of FDS in human into type $1-\mathrm{V}$ and claimed "evolution of this muscle from amphibians to higher primates is the prime reason for the variations".

The present case describes a unique type $\mathrm{V}$ variant observed during cadaveric dissection and this finding will add to note to our knowledge of FDS muscle disparity

\section{Case report}

During undergraduate dissection of a 65-yearold male preserved cadaver by medical students of Faculty of Medicine, University of Jaffna, morphological variation in the FDS was observed on left upper limb. All other forearm muscles were anatomically normal. The FDS had 2 bellies, superficial and deep. The superficial belly had the usual humeroulnar and radial origins, which united to form a single muscle belly. It bifurcated at the region of the upper and middle third of forearm, passed below the flexor retinaculum (Fig 1) and inserted into middle phalanx of $3^{\text {rd }}$ and $4^{\text {th }}$ finger (Fig 3 ). The deep belly originated from medial epicondyle of the humerus (common flexor origin), next to flexor carpi ulnaris muscle. It had double belly appearance (digastric) in upper part of forearm; two muscle bellies connected by an intermediate tendon (Fig 2). The deep belly bifurcated at middle and lower third of forearm. These tendons passed deep to the tendons of superficial belly through carpal tunnel and insert into $2^{\text {nd }}$ and $5^{\text {th }}$ fingers (Fig 3 ). The contralateral forearm does not show any anatomical variation. The median nerve was found deep to the deep belly of FDS.

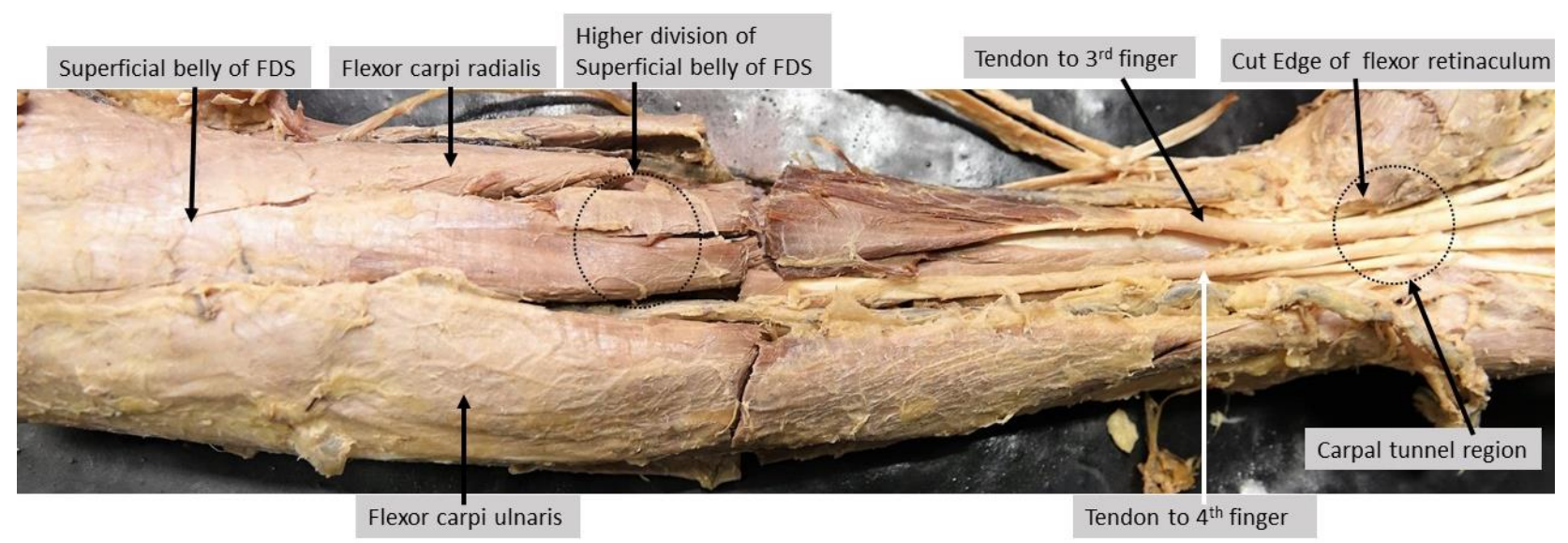

Figure 1: Dissection of the anterior flexor aspect of the forearm showing the superficial belly of FDS dividing again into two bellies nearly at upper and middle third of forearm and tendons of them passing below flexor retinaculam. 
Udhayakumar S, Nilojan JS, Ketheeswaran N, Srishankar S - A unique Anatomical variation of flexor digitorum superficialis muscle: a cadaveric case report

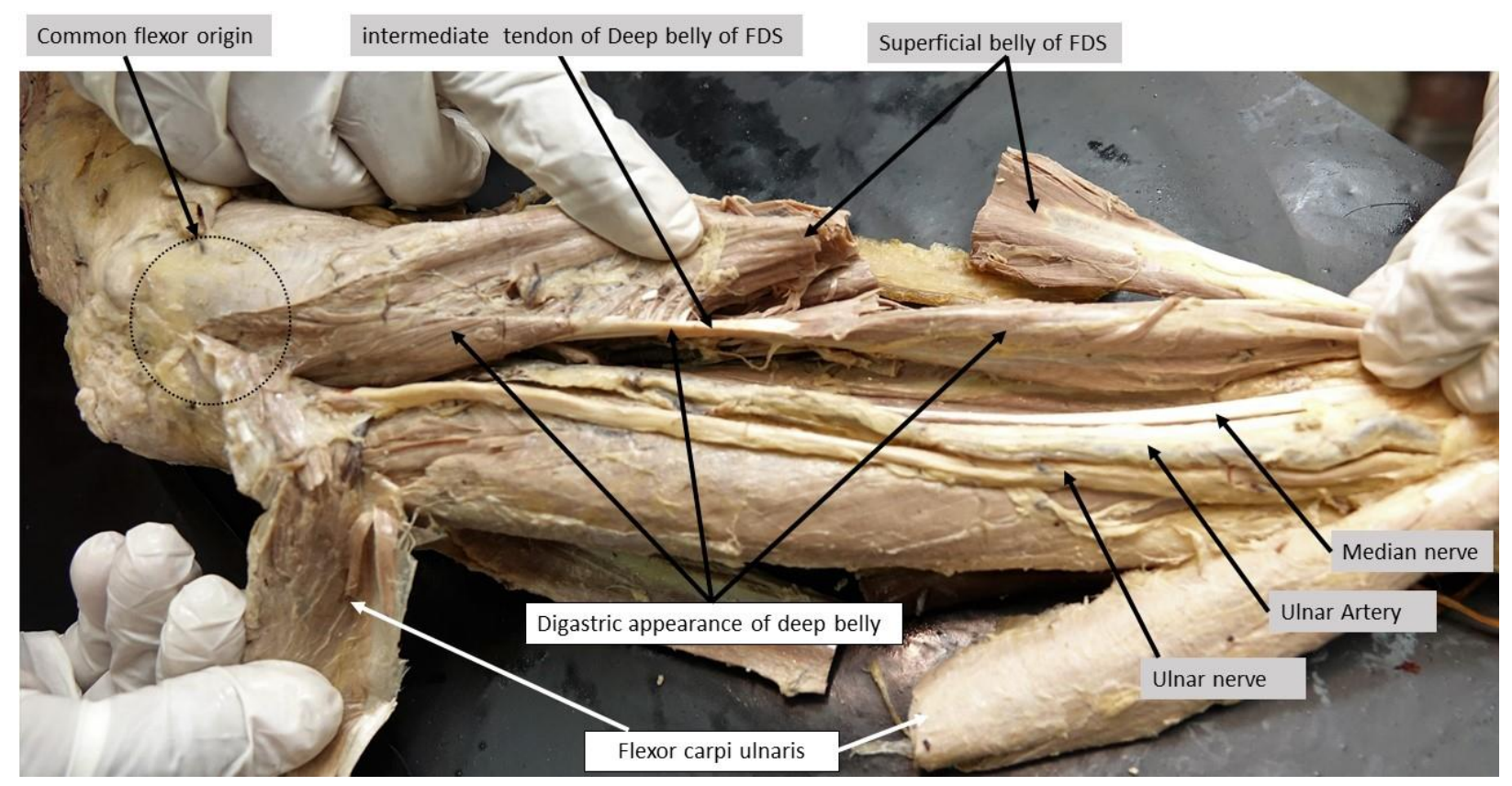

Figure 2: Dissection showing deep belly originating from the common flexor origin and the digastric appearance (double belly appearance) of deep belly in the upper third of forearm

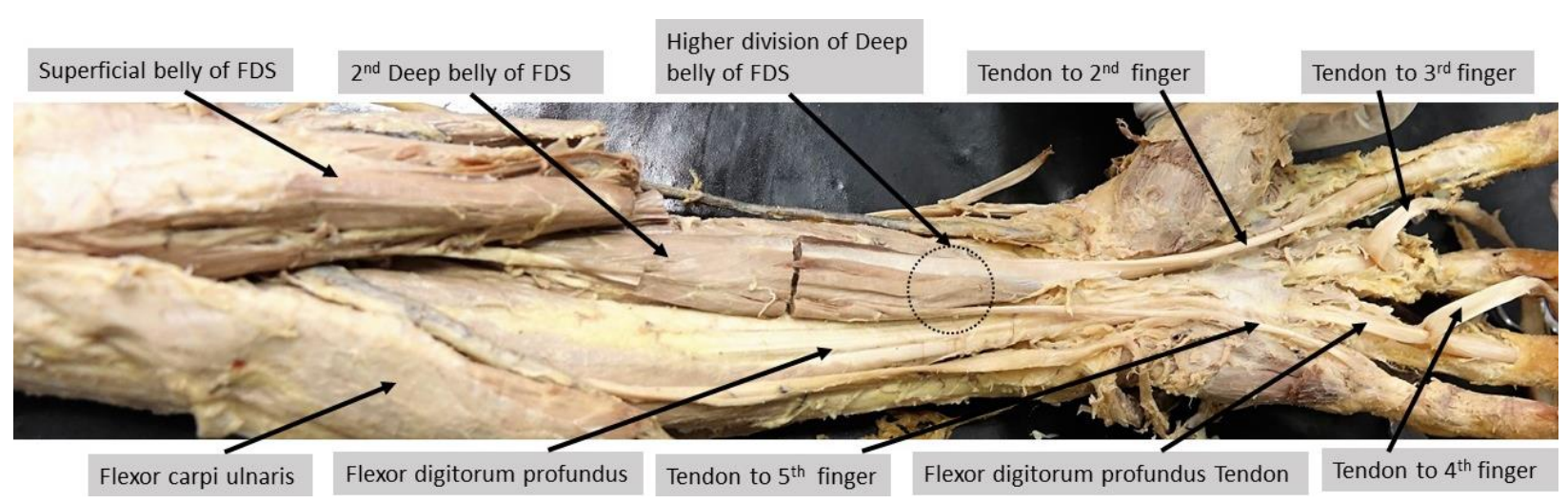

Figure 3: Dissection showing bifurcation of deep belly in the junction of middle and lower third of forearm and insertion of its tendons into $2^{\text {nd }}$ and $5^{\text {th }}$ digits. Insertion of tendons of superficial belly into $3^{\text {rd }}$ and $4^{\text {th }}$ digit can be seen.

\section{Discussion}

Elliot el al (10) reviewed the articles reporting the variations of FDS and they classified the variations of FDS into 5 types. 'Type1 connection between the tendons of the muscle itself; type II - additional muscle belly connection between the FDS tendons and the flexor retinaculum within hand; type III - digastric additional muscle connected to tendon of FDS within hand; type IV - distal extension of muscle belly of forearm towards carpal tunnel and type $\mathrm{V}$ - anatomical variation of FDS muscle in the forearm'. They discuss the development and evolution of FDS. In amphibians flexor muscles of forearm act as wrist flexors and digits are flexed by brevis 
Udhayakumar S, Nilojan JS, Ketheeswaran N, Srishankar S - A unique Anatomical variation of flexor digitorum superficialis muscle: a cadaveric case report

muscles originating within the hand. In mammals these brevis muscles attach with the extrinsic flexor muscle mass evolve from forearm and the digits are flexed by forearm flexor muscles. The brevis muscles become tendons of FDS and FDP passing through the carpal tunnel into the palm. Therefore the abnormal or additional muscle bellies of the FDS in man represent the derangement in the normal process of development or intermediate stages in the development process $(3,10)$.

Tan et al (9) introduced another classification for variations of FDS. The variations are classified into two major categorizes: variation of muscle belly and tendon arrangement. They claimed muscle belly anomalies are mostly incidental findings. When the muscle belly extends to carpal tunnel, it may cause carpal tunnel syndrome. Additional muscle belly in palm may present as a palmar mass. They further added that abnormalities of muscle belly can in the form of brevis type muscle present in the palm or muscle belly extend into carpal tunnel or digastric muscle, where muscle bellies are separated by a tendon in palm. This classification does not take variations in muscle bellies in the forearm into account.

Literature reveal variation of FDS frequently occur in palm of the hand; Type 1I\& III according to Elliot el (10) classification and most frequently anomalies occur in the FDS that serves the index finger $(10,13,14)$. This variant have been reported during surgery as a small mass attached to the middle phalanx of index finger or to the tendon of FDS to index finger proximally and distally (digastric) in the palm. These masses may or may not be painful. Elias and Schulter-Ellis (13) stated that preoperative differential diagnoses of those masses included tendon sheath tumors, lipoma, palmar ganglion, vascular malformations and haematoma. However, Stephens et al (14) emphasized the possibility of an additional muscle belly of FDS should be brought into mind if the mass is soft and becomes firm when proximal interphalangeal joint is flexed against resistance.

Tan et al (9) observed connections between musculotendinous units during clinical examination of 500 subjects. They stated that tendon abnormalities do not produce noticeable symptoms, but they affect evaluation and interpretation of FDS function clinically, especially in a patient with a FDS tendon injury.

Type IV variant, extension of muscular part distally into the carpal tunnel has been observed during carpal tunnel decompression $(10,13)$. Yesilado et al (11) noticed a fleshy muscle belly extending to flexor compartment of wrist without a tendon during forearm surgery. Traction on the muscle caused flexion of proximal interphalangeal joints of medial four fingers.

Type $\mathrm{V}$ variation observed in our study is less common and incompletely described when it is identified in surgery because their dissection is limited to the incision made to treat clinical needs of the case (10). The first case presented by Mainland (2) during cadaveric dissection showed a variant of the FDS where the tendons of the ring and small fingers were connected to three muscles of which two 
Udhayakumar S, Nilojan JS, Ketheeswaran N, Srishankar S - A unique Anatomical variation of flexor digitorum superficialis muscle: a cadaveric case report

additional muscles were in the forearm. In an operation on the flexor aspect of the forearm to remove a lipoma Figueiredo and Hooper (3) identified an anomalous muscle belly lying deep to median nerve in distal forearm and on traction it caused flexion of proximal interphalangeal joint of index finger. But due to limited incision, they couldn't explore the origin of the anomalous muscle belly. Elliot et al (10) identified a variant while performing a surgery, where a small muscle belly originated from deep surface of transverse carpal ligament and attached by a tendon to FDS muscle of middle finger $8 \mathrm{~cm}$ proximal to wrist fold. Nayak et al (4) reported unilateral 2 additional muscle bellies in anterior forearm deep to FDS, originating from it. Radial side one was a Gantzer's muscle ("muscle arises as small belly from forearm flexors and insert either into flexor pollicis longus or FDP") inserting into flexor pollicis longus tendon and the ulnar side belly forming an independent tendon in the forearm, passing through the carpal tunnel. Passive traction on it caused flexion of distal and middle phalanx of middle finger. Rao et al (6) reported a variant during cadaveric dissection, where a separate muscle belly originated from medial epicondyle of humerus and formed a tendon in middle of forearm. The tendon passed deep to the transverse carpal ligament and inserted into middle phalanx of fifth digit. In a series of 100 dissected cadavers Caetano et al (8) presented unilateral variation of FDS in a cadaver, where an accessory digastric muscle belly connected to the FDS in the middle of forearm on its deeper aspect and proximally attached to the common flexor origin. This finding mimic the proximal aspect of the deep belly reported in the present study. Another finding similar to the present report was documented by Shoja et al (5). They observed superficial and two separate fusiform deep muscle bellies. The medial belly originated from common flexor origin and coursed through the distal forearm as a slender and inserted onto the little finger. The radial side belly merged proximally with the deep surface of the superficial part of the FDS and continued distally as a tendon to get inserted onto the index finger. In the present study deep belly wholly originated from medial epicondyle and had a unique morphology in the upper part, 2 bellies and an intermediate tendon.

Morphological variation of muscles of forearm are not common and most of the time they are incidental findings during surgical interventions. These anomalous muscles can produce soft tissue masses and can compress vessels and nerves leading to compression symptoms. Therefore, knowledge of these variation helps the clinicians to detect he etiology, interpret symptoms and to manage the patients with such anomalous presentation.

Brandsma et al (15) pointed out another importance of knowing the FDS variant. Following injury to ulnar and radial nerve, flexor digitorum superficialis tendons are used in tendon transfer to correct claw hand deformity and loss of opposition of the thumb respectively. Variations in the origin and course of the tendon may cause difficulty in identifying these tendons during surgical procedures, especially when window incisions are made over the normal orientation of these tendons. 
Udhayakumar S, Nilojan JS, Ketheeswaran N, Srishankar S - A unique Anatomical variation of flexor digitorum superficialis muscle: a cadaveric case report

Identifying these variations will add knowledge on morphological variation of FDS and knowledge of the anatomical variations help to understand atypical findings on physical examination and during surgery and help to interpret abnormal radiological findings.

\section{Acknowledgement}

Authors thank the Medical Education and Audiovisual Unit of Faculty of Medicine, University of Jaffna for their assistance in taking photographs

\section{Corresponding author}

Sivananthini Udhayakumar,

Department of Anatomy,

Faculty of Medicine,

University of Jaffna, Sri Lanka.

Postal code 40000

E-mail: nanthini@univ.jfn.ac.lk

Tel: +940779983012

Fax: 0212222073

\section{BY 4.0}

This is an Open Access article distributed under the terms of the Creative Commons Attribution 4.0 license (unless stated otherwise) which permits unrestricted use, distribution and reproduction in any medium, provided the original work is properly cited. Copyright is retained by the author(s).

\section{References}

1. Standring S. Gray's anatomy: the anatomical basis of clinical practice. $39^{\text {th }}$ ed. Edinburgh: Elsevier Churchill Livingstone Publishers : 2006.

2. Mainland D: An uncommon abnormality of the flexor digitorum sublimis muscle. $\mathrm{J}$ Anat 1927; $62: 86-9$,

3. Figueiredo UM, Hooper G. Abnormal course of the median nerve associated with an anomalous belly of flexor digitorum superficialis. Hand 1980; 12 : $273-4$

4. Nayak SR, Ramanathan L, Prabhu LV, Raju S. Additional flexor muscles of the forearm: case report and clinical significance. Singapore Med J. 2007; 48 (8) : $231-3$

5. Shoya MM, Tubbs RS, Loukas M, Shokouhi G. The split flexor digitorum superficialis. Ital J Anat Embryol, 2008; 13 (2) : $103-7$

6. Rao M, Aswieni LS, Somayaji NS, Mishra S, Guru A, Rao A. Separate belly and tendon of flexor digitorum superficialis to the fifth digit. Oman Med J. 2011; 26 (6). DOI 10, 5001/omj.2011.120

7. Anita T, Kalbande S, Asha K, Dombe D, Jayasree N. A unique variation of flexor digitorum superficialis muscle and its clinical significance. J Life Sci. 2012; 4 (1) : $39-43$

8. Caetano EB, Neto JJS, Ribas LAA, Milanello EV. Accessory muscle of the flexor digitorum superficialis and its 
Udhayakumar S, Nilojan JS, Ketheeswaran N, Srishankar S - A unique Anatomical variation of flexor digitorum superficialis muscle: a cadaveric case report

clinical implications. Rev Bras Ortop.

2017; 52 (6) : $731-4$

9. Tan JS, Oh L, Louis DS. Variations of the flexor digitorum superficialis as determined by an expanded clinical examination. J Hand Surg Am 2009; 34 :900 - 6 .

10. Elliot D, Khandwala AR, Kulkami M Anomalies of the flexor digitorum superficialis muscle. J Hand Surg Br 1999; $24: 570-4$

11. Yesilada AK, Tathdede HS, Cakmak E, Kryak MV, Krhnc L. Anomalous large unique muscle belly of flexor digitorum superficialis and the absence of palmaris longus in th forearm. Journal of plastic, Reconstre \& Aesthetic surg. 2013; $66: 137$ -9 .

12. Still JM, Kleinert HE. Anomalous muscles and nerve entrapment in the wrist and hand. Plastic Reconstr Surg. 1973; 52 (4) : $394-400$
13. Elias LS and Schulter-Ellias FP 1985 Anomalous flexor superficialis indicis: two case reports and literature review J Hand surg Am. 1985; 10 (2) 296 - 9

14. Stephens N, Marques E, Livingston C. Anomalous flexor digitorum superficialis muscle belly presenting as a mass within the palm, Can J Plast Surg. 2007; 15 (1): 44- 6.

15. Brandsma JW, Ottenhoff-de jonge MW. Flexor Digitorum Superficialis Tendon Transfer for Intrinsic Replacement: Longterm results and the effect on donor fingers J Hand surg Eu. 1992; 10.1016/02667681(92)90187-7 